\title{
CGRP antibody therapy in patients with drug resistant migraine and chronic daily headache: a real-world experience
}

\author{
Armin Scheffler ${ }^{1 *}$, Hannah Schenk', Sebastian Wurthmann ${ }^{1}$, Michael Nsaka ${ }^{1}$, Christoph Kleinschnitz ${ }^{1,2}$,
} Martin Glas ${ }^{2}$ and Dagny Holle ${ }^{1}$

\begin{abstract}
Background: Calcitonin gene-related peptide (CGRP) (receptor) antibodies (erenumab, fremanezumab and galcanezumab) are increasingly used in prophylactic treatment of migraine. In the approval studies, severely affected patients with migraine and chronic daily headache without any headache free days were excluded. Thus, less is known about the effectiveness of CGRP antibody treatment in this cohort.

Methods: Clinical routine data of 32 patients with migraine and daily headache were analysed after three months of treatment with a CGRP antibody (16 erenumab, 7 galcanezumab, 9 fremanezumab), including changes of monthly headache days (MHD) monthly migraine days (MMD) and monthly acute medication intake (AMD) as well as migraine characteristics. Statistical analysis was performed with the Wilcoxon-Test. Migraine characteristics were analysed descriptively.

Results: The number of MHD was significantly reduced (mean reduction (standard error), p-value): (-4.2 (1.3), $p=$ $0.009)$ as well as MMD (-4.3 (1.6), $p=0.033)$. Four patients $(13 \%)$ reached a $50 \%$ reduction regarding MHD and 8 patients $(25 \%)$ regarding MMD, migraine duration and intensity improved under therapy.

Conclusions: Despite the low responder rate, CGRP antibodies can be effective at least in a few cases of severely affected patients with drug resistant migraine and chronic daily headache.
\end{abstract}

Trial registration: Retrospective registered.

Keywords: Migraine, CGRP antibody, Therapy, Real-world, Chronic daily headache

\footnotetext{
*Correspondence: armin.scheffler@uk-essen.de

'Department of Neurology and Center for Translational Neuro- and Behavioral Sciences (C-TNBS), West German Headache Center, University Hospital Essen, University Duisburg-Essen, Hufelandstr. 55, 45147 Essen, Germany

Full list of author information is available at the end of the article
}

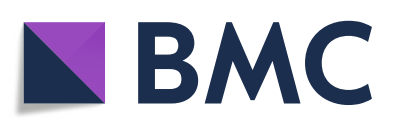

(- The Author(s). 2021 Open Access This article is licensed under a Creative Commons Attribution 4.0 International License, which permits use, sharing, adaptation, distribution and reproduction in any medium or format, as long as you give appropriate credit to the original author(s) and the source, provide a link to the Creative Commons licence, and indicate if changes were made. The images or other third party material in this article are included in the article's Creative Commons licence, unless indicated otherwise in a credit line to the material. If material is not included in the article's Creative Commons licence and your intended use is not permitted by statutory regulation or exceeds the permitted use, you will need to obtain permission directly from the copyright holder. To view a copy of this licence, visit http://creativecommons.org/licenses/by/4.0/. The Creative Commons Public Domain Dedication waiver (http://creativecommons.org/publicdomain/zero/1.0/) applies to the data made available in this article, unless otherwise stated in a credit line to the data. 


\section{Background}

Monoclonal calcitonin gene-related peptide (CGRP) antibodies (in Europe erenumab, fremanezumab and galcanezumab) are being used more often in the prophylactic therapy of migraine. First real-world experiences and open label studies seem to confirm the benefit of the approval studies [1-3]. Nevertheless, all protocols of the approval studies excluded or had at least strong limitations regarding the severely affected patients with chronic daily headache $(\mathrm{CDH})$ without any headache free days [4-6]. These patients were excluded from the trials primarily due to the expected poor response to therapy. Other studies showed a limited response to the pre-existing migraine prophylactic drugs regarding $\mathrm{CDH}[7,8]$.

So far, no clinical data about the potential benefit of CGRP antibody therapy in this cohort are available. However, new therapeutic options are needed as these patients suffer more than others from migraine. We analysed the therapeutic effectiveness of CGRP antibodies in patients with migraine and daily headache after 3 months of treatment.

\section{Methods}

We retrospectively analysed routine clinical data of 32 patients with migraine presented at the West German Headache Center, Department of Neurology, University Hospital Essen, Germany between November 2018 and July 2021. The analysis was approved by the independent ethics committee of the University Hospital Essen (199004-BO). All patients gave written consent to general analysis of their personal and clinical data. Patients meeting the following criteria: (a) Fulfilled criteria for migraine (ICHD-3) (b) $\mathrm{CDH}$ with headache every in day the last 90 days before treatment. (c) completion of a 90 days treatment interval with erenumab (16 patients, $70 \mathrm{mg} / \mathrm{month}$ ), galcanezumab (7 patients) or fremanezumab (9 patients, $225 \mathrm{mg} /$ month or $675 \mathrm{mg} / 3$ months)). Three patients had already received erenumab in the past. Patients treated with the three $\mathrm{mAB}$ were pooled for later statistical analysis. Clinical data reported by patients were compared to a paper-based or electronical headache diary. Monthly headache days (MHD) and monthly migraine days (MMD) were defined as the average monthly mean values over the respective total observation period of 90 days. A headache day was defined as a day with any kind of headache, a migraine day was defined by patients when they had severe pain, migraine pain characteristics (pulsating, one-sided pain), aura symptoms, vegetative symptoms like phono- or photophobia, nausea, vomiting, need for rest, or when triptans were taken. A 50 and $30 \%$ responder rate were defined as a reduction by at least 50 or $30 \%$ over the three months of treatment. Most patients answered questionnaires regarding different aspects of migraine: intensity of migraine $(n=$ $32)$, duration of the migraine attack $(n=32)$, effect of acute therapy $(n=30)$, effect on the aura $(n=31)$, need for rest $(n=31)$, dizziness $(n=30)$, nausea $(n=31)$, phono- and photophobia $(n=31)$ as well as therapy satisfaction $(n=32)$. Data about changes of AMD was available of $n=30$ patients. Due to reasons of reimbursement by the German statutory health insurance, all treated patients had tried six approved prophylactic drugs previously without sufficient treatment effects, had discontinued those due to side effects or were not eligible for intake due to contraindication. Approved drug classes were the following: betablockers (metoprolol or propranolol), tricyclic antidepressants (amitriptyline), calcium channel blockers (flunarizine), anticonvulsants (topiramate and valproic acid) and onabotulinumtoxin A. If there was a preexisiting migraine modulating comedication, medication was not altered. Data were analysed using SPSS software (IBM SPSS Statistics for Windows, Version 27.0. Armonk, NY, USA, IBM Corp), RStudio (Version 1.4, Boston, MA, USA, RStudio PBC) and Excel 2019 (Version 1809, Redmond, Washington, USA, Microsoft). Wilcoxon's test was used to compare MHD, MMD and AMD before and after treatment. Bonferoni's method for multiple comparisons was set (two-tailed $p$-value*3, alpha $=0.05)$. For statistical analysis of differences in response for MHD and MMD among the respective antibodies the Kruskal-Wallis-Test was used. Patient reported outcomes were analysed descriptively. (A part of the methods has already been used elsewhere [2]).

\section{Results}

Clinical data of 32 patients with migraine and $\mathrm{CDH}$ were analysed. Details regarding demographic data, aura and duration of the disease, comedication and statistics about MMD, MHD and AMD before and after treatment are summarized in Table 1 (Table 1 here).

After 3 months of treatment a significant reduction of MHD and MMD was observed. Regarding medication overuse headache $(\mathrm{MOH}), 40 \%(n=12$ of 30) before and $17 \%(n=5$ of 30$)$ after treatment had more than 10 acute medication intake days per month. However, no significant reduction of AMD was reached due to therapy after alpha adjustment (Fig. 1). Whereas 11 patients had any response to the treatment regarding daily headache, 21 patients showed no effect regarding MHD. $50 \%$ responder rate of MHD was $13 \%(n=4)$ and $30 \%$ responder rate was $22 \%(n=7)$. Regarding MMD, $50 \%$ responder rate was $25 \%(n=8)$ and $30 \%$ responder rate was $41 \%(n=13)$. There was no significant difference in the changes of MHD or MMD after three months between the three mAB (Kruskal-Wallis test: $p=0.767$ and 0.813 , respectively). 
Table 1 Patients' characteristics and statistics. a) Patients characteristics, antibody therapy and comedication. A comedication of several medication was possible. Comedication: A: amitriptyline, B: betablocker, T: topiramate, V: valproic acid, F: flunarizine, others: magnesium and other antidepressive drugs like selective serotonine reuptake inhibitors). b) changes in monthly headache days (MHD) and monthly migraine days (MMD) and monthly acute medication intake (AMD) after 3 months of treatment after the respective CGRP antibody therapie. MHD and MMD were significantly reduced after 3 months of treatment

\begin{tabular}{|c|c|c|}
\hline a) & male/female & $8 / 24$ \\
\hline age & mean value (min/max) & $42.5(19 / 77)$ \\
\hline aura & existing & 17 \\
\hline \multirow{3}{*}{ duration of migraine in y } & $\begin{array}{c}\text { patients with a clear onset; } \\
\text { mean value (SD) }\end{array}$ & $\begin{array}{c}\mathrm{n}=25 ; \\
22.3(13.3)\end{array}$ \\
\hline \multirow{3}{*}{ antibody } & erenumab & 16 \\
\cline { 2 - 3 } & galcanezumab & 7 \\
\cline { 2 - 3 } & fremanezumab & 9 \\
\hline \multirow{3}{*}{ comedication } & patients with comedication & 17 \\
\cline { 2 - 3 } & at least one of $A, B, T, V, F$ & 11 \\
\cline { 2 - 3 } & at least one of other & 6 \\
\hline
\end{tabular}

b)

\begin{tabular}{|c|c|c|c|}
\hline & $\mathbf{n}$ & $\begin{array}{c}\text { reduction after 3 months } \\
\text { mean value (SE) }\end{array}$ & p-value (adjusted) \\
\hline MHD & 32 & $-4.2(1.3)$ & 0.009 \\
\hline MMD & 32 & $-4.3(1.6)$ & 0.033 \\
\hline AMD & 30 & $-2.4(1.3)$ & 0.123 \\
\hline
\end{tabular}

The improvement of migraine characteristics was reported, in particular an improvement of migraine intensity and duration, nausea as well as acute drug effect. No significant improvement was observed with regard to other clinical characteristics (Fig. 2).

Forty-one $\%$ of patients $(n=13)$ were very satisfied or satisfied with the therapy, $44 \%(n=14)$ were moderately satisfied, $16 \% \quad(n=5)$ were unsatisfied or very unsatisfied.

Side effects were reported by $38 \%(n=12)$ of patients, none of the patients reported a severe side effect. Main side effects were constipation with $21 \%$ and nausea/ vomiting with $16 \%$ of all the reported side effects $(n=4$ and $n=3$, respectively).

\section{Discussion}

Our real-world data shows the effectiveness and tolerability of CGRP antibody treatment in drug resistant patients with migraine and headache every day. Chronic daily headache $(\mathrm{CDH})$ is often defined as the chronic form of headache diseases with at least 15 headache days per month [9]. Different mechanisms like acute medication overuse, neurotransmitter pathway modulations or alteration of pain related brain structures during a long burden of headache disease are discussed in pathophysiology of CDH [10].
The pivotal studies of the several CGRP antibodies focused on MMD reduction as the primary endpoint at different time points (50\% responder rate: galcanezumab: $27.6 \%$ (120 mg/month) and $27.5 \%$ (240 mg/month) [11]; erenumab $40 \%$ (70 mg/month) and $41 \%$ (140 mg/month) [4]; fremanezumab $38 \%$ (675 mg/3 months) and $41 \%$ (225 mg/month) [5]). Our study showed a low $50 \%$ responder rate in MHD $(13 \%, n=4)$ and a better response in MMD $(25 \%, n=8)$. However, the pivotal studies did not include patients with daily headache. Little data are available for other approved licensed migraine prophylactic drugs used in $\mathrm{CDH}$ therapy and the existing data shows a limited effect. In a study focused on amitriptyline, the $50 \%$ responder rate of MHD in a subgroup analysis of $\mathrm{CDH}$ (defined as more than $17 \mathrm{MHD}$ ) was $25 \%$ after four weeks of treatment $(n=9$ of 36$)$ with significant results compared to placebo $(p=0.043)$, but no significant difference to placebo was observed after 8 weeks of treatment [7]. The treatment of patients with $\mathrm{CDH}$ (defined as headache every day) with onabotulinumtoxin A showed a $50 \%$ MHD responder rate of $17.0 \%$ $(n=18$ of 106$)$ from baseline at week 24 and $39.6 \%$ ( $n=19$ of 48$)$ at week 108 . However, only less than half of the patients participated by week $108(n=48)$ compared to week $24(n=106)$, so the responder rate may be biased to the last timepoint [8]. 


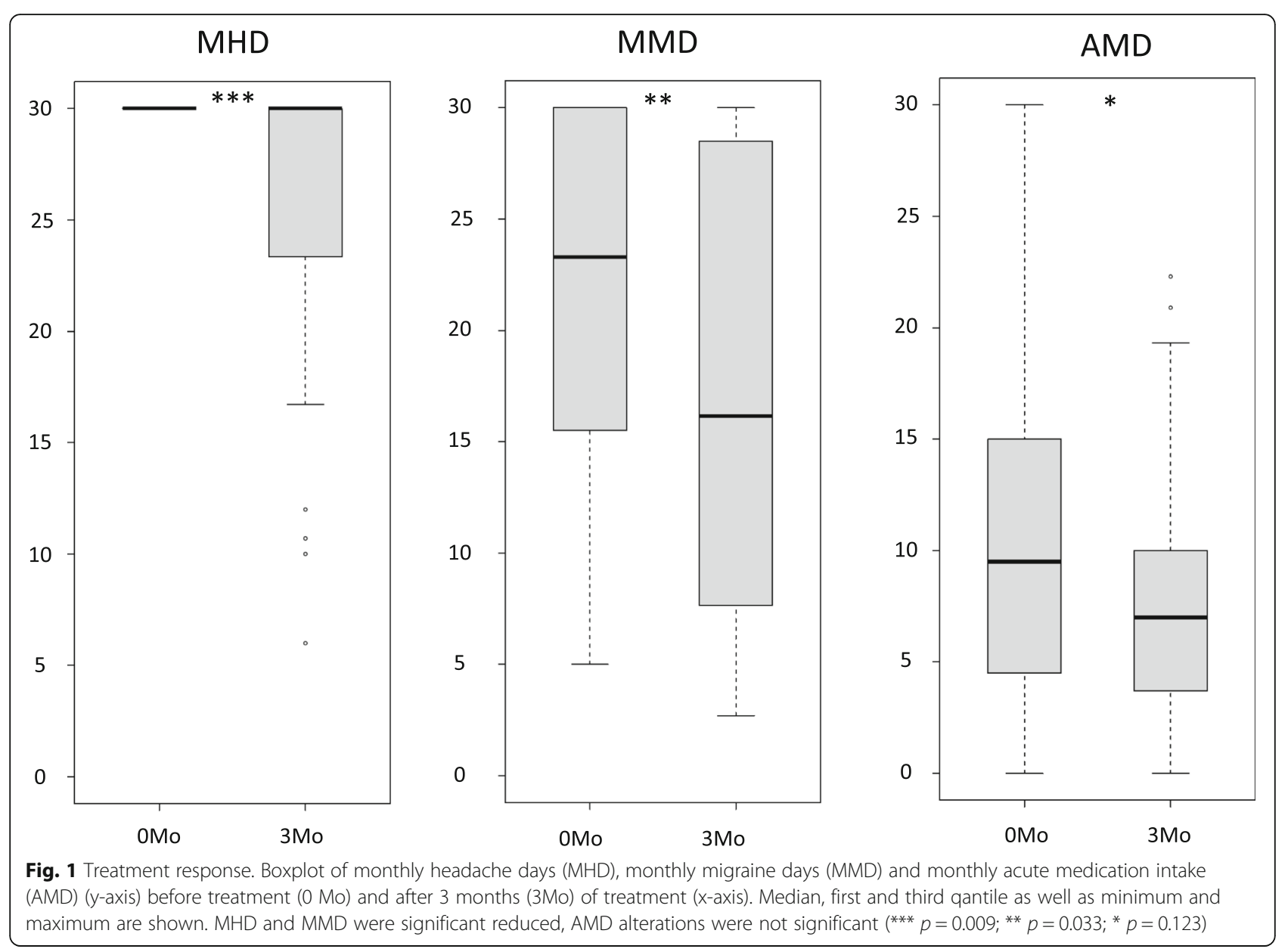

Nevertheless, our data also shows a good response in MHD in at least these four patients and in 8 patients regarding MMD who previously failed all other first line therapies or were not able to receive them. Despite the low responder rate, patient reported better satisfaction than the actual reduction would lead on to expect. A possible reason is the observed improvement of the migraine characteristics, especially in duration and intensity of the migraine attack. Tolerability was good, and side effects were mild and comparable to previous CGRP mAb studies $[1-3,12]$.

There are some limitations of our study. First, patients who are affected with drug resistant migraine and $\mathrm{CDH}$ are rare and we decided to pool the data of all antibodies for statistical analysis. Thus, differences in the respective antibodies, e.g. better or worse effect cannot be identified. Nevertheless, considering the small number of cases for each antibody at least no significant difference between the respective antibody was detected. Furthermore, we have only retrospective real-world data and no placebo group. Great expectations in CGRP antibody therapy as a new and modern treatment option could feign an improvement. The long-term effect is not known. Further studies have to confirm the first impressions, that CGRP mAB could be beneficial to at least a few of these severely affected patients.

Although side effects were mild, one patient discontinued the therapy after 2 months of treatment because of symptoms of cold and allodynia of the scalp. Thus, the patient was not included to the analysis due to the incomplete treatment interval. In principle, a false positive therapy effect is therefore possible.

Another limitation is the differentiation between headache and migraine days in this special cohort. Patients with migraine and daily headache often suffer from migraine characterised pain and symptoms every day (e.g. phono- or photophobia, one sided headache) and only feel a worsening of the already existing symptoms in a migraine attack, making it difficult to distinguish between MHD and MMD. Thus, both parameters should be focused quantify the therapy effect. Especially headache-free days should be a target of the treatment of patients with $\mathrm{CDH}$. 


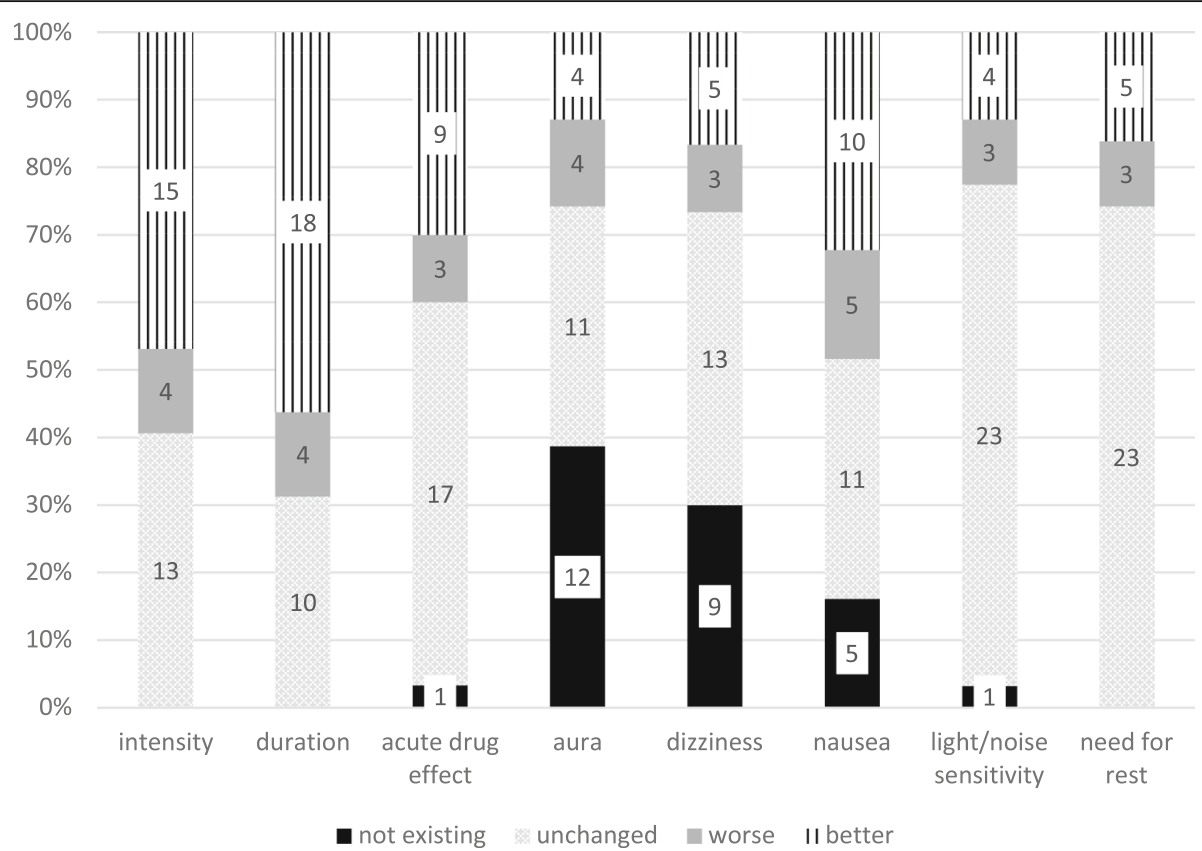

Fig. 2 Migraine characteristics. Changes of migraine characteristics after three months of treatment. The relative proportion and the absolute number of patients who noticed the respective change are noted. All non-existent, non-applicable and non-occurring events are summarised under not existing

Regarding acute drug intake, 7 patients had no longer acute drug medication intake over 10 days a month after 3 months of treatment. This needs to be investigated in further studies, as due to the lack of significant change of AMD, this may be purely coincidental.

\section{Conclusions}

Our data shows that CGRP antibodies could be a possible therapy option for at least a few severly affected patients who failed all previous therapies. Thus, a therapy trial may be useful, because a therapy response and an improvement of other migraine symptoms seems possible in this cohort. Further studies are needed to confirm longterm effects with a larger number of patients.

\section{Abbreviations}

AMD: monthly acute medication intake; CGRP: calcitonin gene-related peptide; $\mathrm{CDH}$ : chronic daily headache; ICHD-3: International headache classification; mAB: monoclonal antibody; MHD: monthly headache days; MMD: monthly migraine days; $\mathrm{MOH}$ : medication overuse headache: SE: standard error

\section{Acknowledgements}

Benjamin Kubo corrected the manuscript regarding language. The study is part of the doctor thesis of one of the authors (HS).

\section{Authors' contributions}

AS and HS had the major role in the acquisition of data. AS analysed and interpreted the data and drafted the manuscript. HS analysed and interpreted the data and revised the manuscript for intellectual content. SW, $M N, C K$, and MG interpreted the data and revised the manuscript for intellectual content. DH designed and conceptualized the study, interpreted the data and drafted the manuscript. All authors read and approved the final manuscript.
Funding

No funding. Open Access funding enabled and organized by Projekt DEAL.

\section{Availability of data and materials}

The datasets used and/or analysed during the current study are available from the corresponding author on reasonable request.

\section{Declarations}

Ethics approval and consent to participate

The analysis was approved by the independent ethics committee of the University Hospital Essen (19-9004-BO) and all patients gave written consent to general analysis of their personal and clinical data.

\section{Consent for publication}

Not applicable.

\section{Competing interests}

Martin Glas received honoraria from Novartis, UCB, Teva, Bayer, Novocure, Medac, Merck, Kyowa Kirin, has a consulting or advisory role to declare for Roche, Novartis, AbbVie, Novocure, and Daiichi Synkyo, and received travel fees from Novocure and Medac.

Dagny Holle has received scientific support and/or honoraria from Biogen, Novartis, Lilly, Sanofi-Aventis, Teva, Allergan, Hormosan.

Christoph Kleinschnitz has received honoraria, a consulting or advisory role to declare for Alexion, Almirall, Amgen, Amicus, Bayer, Biogen, Biotronik, Boehringer Ingelheim, Bristol Myers-Squibb, Celgene, CSL Behring, Daiichi Sankyo, Desitin, Eisai, Ever Pharma, GE Healthcare, MedDay Pharmaceuticals, Merck Serono, Mylan, Novartis, Pfizer, Roche, Sanofi-Genzyme, Siemens,

Stago, Teva.

Michael Nsaka received travel fees from Licher MT.

Armin Scheffler received travel fees from Teva, honoraria for participation on an advisory board of Novartis.

Sebastian Wurthmann reports personal fees from Allergan, personal fees from Teva, personal fees from Novartis, outside the submitted work. Hannah Schenk declares that there is no conflict of interest. 


\section{Author details}

'Department of Neurology and Center for Translational Neuro- and Behavioral Sciences (C-TNBS), West German Headache Center, University Hospital Essen, University Duisburg-Essen, Hufelandstr. 55, 45147 Essen, Germany. ${ }^{2}$ Department of Neurology and Center for Translational Neuro- and Behavioral Sciences (C-TNBS), Division of Clinical Neurooncology, University Hospital Essen, University Duisburg-Essen, Hufelandstr. 55, 45147 Essen, Germany.

Received: 19 July 2021 Accepted: 6 September 2021

Published online: 20 September 2021

\section{References}

1. Ornello R, Casalena A, Frattale I, Gabriele A, Affaitati G, Giamberardino MA et al (2020) Real-life data on the efficacy and safety of erenumab in the Abruzzo region, central Italy. J Headache Pain 21(1):32

2. Scheffler A, Messel O, Wurthmann S, Nsaka M, Kleinschnitz C, Glas M et al (2020) Erenumab in highly therapy-refractory migraine patients: First German real-world evidence. J Headache Pain 21(1):84

3. Camporeale A, Kudrow D, Sides R, Wang S, Van Dycke A, Selzler KJ et al (2018) A phase 3, long-term, open-label safety study of Galcanezumab in patients with migraine. BMC Neurol 18(1):188

4. Tepper S, Ashina M, Reuter U, Brandes JL, Doležil D, Silberstein S et al (2017) Safety and efficacy of erenumab for preventive treatment of chronic migraine: a randomised, double-blind, placebo-controlled phase 2 trial. Lancet Neurol 16(6):425-434

5. Silberstein SD, Dodick DW, Bigal ME, Yeung PP, Goadsby PJ, Blankenbiller T et al (2017) Fremanezumab for the Preventive Treatment of Chronic Migraine. N Engl J Med 377(22):2113-2122

6. Detke HC, Goadsby PJ, Wang S, Friedman DI, Selzler KJ, Aurora SK (2018) Galcanezumab in chronic migraine: The randomized, double-blind, placebocontrolled REGAIN study. Neurology. 91(24):e2211-21

7. Couch JR, for the Amitriptyline Versus Placebo Study Group (2011) Amitriptyline in the Prophylactic Treatment of Migraine and Chronic Daily Headache. Headache J Head Face Pain 51(1):33-51

8. Dodick DW, Mauskop A, Elkind AH, DeGryse R, Brin MF, Silberstein SD et al (2005) Botulinum Toxin Type A for the Prophylaxis of Chronic Daily Headache: Subgroup Analysis of Patients Not Receiving Other Prophylactic Medications: A Randomized Double-Blind, Placebo-Controlled Study. Headache J Head Face Pain 45(4):315-324

9. Silberstein SD (2005) Chronic daily headache. J Am Osteopath Assoc 105(4 Suppl 2):23S-29S

10. Welch MKA, Goadsby PJ (2002) Chronic daily headache: nosology and pathophysiology: Curr Opin Neurol. 15(3):287-95

11. Ruff DD, Ford JH, Tockhorn-Heidenreich A, Sexson M, Govindan S, Pearlman EM et al (2019) Efficacy of galcanezumab in patients with chronic migraine and a history of preventive treatment failure. Cephalalgia 39(8):931-944

12. Goadsby PJ, Silberstein SD, Yeung PP, Cohen JM, Ning X, Yang R et al (2020) Long-term safety, tolerability, and efficacy of fremanezumab in migraine: A randomized study. Neurology 95(18):e2487-e2499

\section{Publisher's Note}

Springer Nature remains neutral with regard to jurisdictional claims in published maps and institutional affiliations.

Ready to submit your research? Choose BMC and benefit from:
- fast, convenient online submission
- thorough peer review by experienced researchers in your field
- rapid publication on acceptance
- support for research data, including large and complex data types
- gold Open Access which fosters wider collaboration and increased citations
- maximum visibility for your research: over 100M website views per year
At BMC, research is always in progress.
Learn more biomedcentral.com/submissions

